\title{
Coccidioides immitis: Two cases of misidentified mycosis
}

\author{
Christine H Lee MD FRCPC ${ }^{1,2}$, Lindsay Wilcox ART ${ }^{2}$, Katherine Chorneyko MD FRCPC ${ }^{1,2}$, Andrew Mclvor MD FRCPC 3
}

\begin{abstract}
CH Lee, L Wilcox, K Chorneyko, A McIvor. Coccidioides immitis: Two cases of misidentified mycosis. Can Respir J 2008;15(7):377-379.
\end{abstract}

In the present report, two cases of pulmonary coccidioidomycosis that caused a diagnostic confusion are presented. The first case was initially misdiagnosed as nonsmall cell carcinoma, and both cases were initially misidentified as blastomycosis because of the presence of an atypical morphological form of Coccidioides immitis.

\section{Coccidioides immitis : Deux cas de mycose mal identifiés}

On présente dans ce rapport deux cas de coccidioïdomycose pulmonaire ayant prêté à une confusion diagnostique. Le premier cas a d'abord été diagnostiqué à tort comme un cancer non à petites cellules et les deux cas ont, à tort, été identifiés initialement comme une blastomycose en raison de la morphologie atypique de Coccidioides immitis.

Key Words: Coccidioides immitis; Pulmonary mycosis

Coccidioides immitis exists in the semi-arid desert of the Usouthwestern United States, northern Mexico, and Central and South America. An estimated 100,000 new cases of $\mathrm{C}$ immitis infection occur in the United States annually, and most commonly involve the lungs (1). Recognition of pulmonary infection is guided by an understanding of the epidemiological factors associated with exposure, the use of appropriate diagnostic tests and an awareness of the diverse manifestations of the pathogen $(1,2)$.

$\mathrm{C}$ immitis is a dimorphic fungus that exhibits morphological variation in its mycelial and parasitic forms. It exists in the desert soil as a mold. As the cells degenerate, barrelshaped arthroconidia break off from the hyphae and become airborne. Arthroconidia undergo significant morphological changes following inhalation into the lung. Within living tissue, it typically swells and develops into a large spherule containing numerous endospores. Endospores are released from ruptured spherules and develop additional spherules in the infected host. Repeated cycles of endospore release and spherule formation in the lung lead to an area of pneumonitis; diagnosis is usually determined based on the presence of these endospores $(1,3,4)$.

In rare cases, $\mathrm{C}$ immitis has been observed to have severe reactive changes in bronchial epithelial cells as well as uncharacteristic hyphae, which form arthroconidia in tissues or air spaces in the lung (2). Because of the presence of these uncharacteristic hyphae, reactive bronchial epithelial cells in pulmonary coccidioidomycosis have been misdiagnosed as carcinoma in cytological examinations $(4,5)$. Finally, there have also been reports of immature, atypical spherules of $\mathrm{C}$ immitis, which have been confused with the budding cells of Blastomyces dermatitidis (2). The presence of these unusual morphological forms can lead to an incorrect diagnosis.

\section{CASE PRESENTATIONS}

\section{Case 1}

A 72-year-old man presented with a four-month history of progressive dyspnea, hemoptysis and weight loss. The patient was a resident of southwestern Ontario and had been travelling to Arizona annually for six years. He had a significant smoking history of 80 pack-years. Three years previously, he had a left upper lobe cavitary lung lesion diagnosed as a poorly differentiated bronchogenic carcinoma with coexistent blastomycosis. For treatment, a left upper lobectomy was performed and itraconazole was given for six months. One year before his current presentation, he had received an additional 12 -month course of itraconazole therapy for recurrent pulmonary blastomycosis.

The patient's most recent chest x-ray showed left pleural thickening, unchanged from three years previously. A presumptive diagnosis of recurrent bronchogenic carcinoma and/or blastomycosis was made, and the patient underwent investigation with fibre optic bronchoscopy.

On bronchoscopy, abnormal tissue was found invading the left lower lobe. Bronchoalveolar lavage (BAL), along with brushings and a biopsy, was performed. The bronchial washing smear showed atypical cells, interpreted as a poorly differentiated nonsmall cell carcinoma along with occasional organisms identified as Blastomyces species. The biopsy specimen showed atypical squamous epithelium and fungal organisms compatible with Blastomyces species, but no malignant cells were detected. Direct microscopy of the tissue biopsy, which was performed in the hospital microbiology laboratory using a calcofluor white preparation, showed the presence of a few spherules, suggestive of $\mathrm{C}$ immitis. A culture of the specimen grew $\mathrm{C}$ immitis. A nucleic acid probe (AccuProbe, Gen-Probe Inc, USA) further confirmed that the isolate was $\mathrm{C}$ immitis.

\footnotetext{
${ }^{1}$ Department of Pathology and Molecular Medicine; ${ }^{2}$ Hamilton Regional Laboratory Medicine; ${ }^{3}$ Department of Medicine, McMaster University, Hamilton, Ontario

Correspondence: Dr Christine H Lee, Department of Pathology and Molecular Medicine, McMaster University, Hamilton Regional Laboratory Medicine, St Joseph's Healthcare, 50 Charlton Avenue East, 424 Luke Wing, Hamilton, Ontario L8N 4A6. Telephone 905-521-6021, fax 905-521-6083, e-mail clee@mcmaster.ca
} 


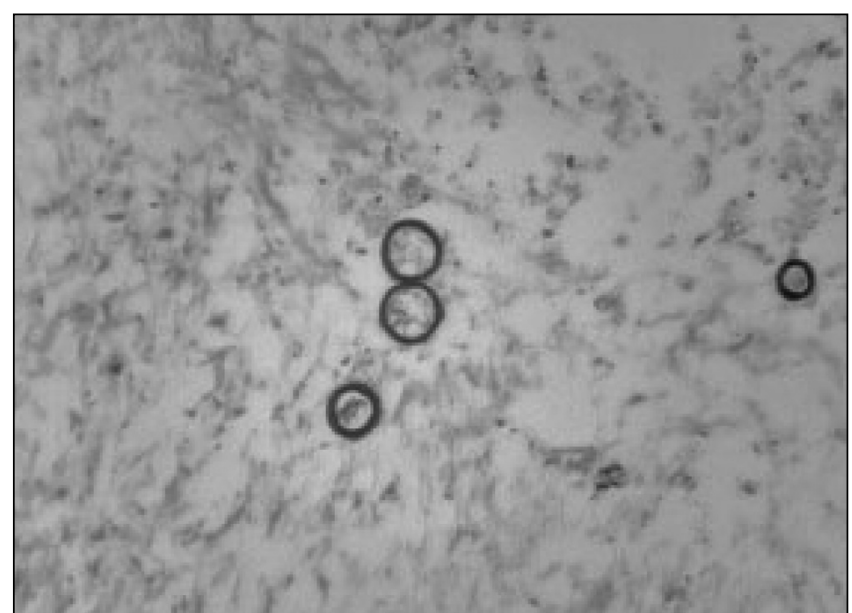

Figure 1) A bronchoalveolar lavage specimen stained with Gomori methenamine silver

In light of the alternative diagnosis, the microscopic slides of the lung specimens obtained previously were re-examined. Periodic acid-Schiff and Gomori methenamine silver stains showed immature nonendosporulating cells and semi-mature spherules consistent with Coccidioides species (Figure 1). Typical mature spherules of $\mathrm{C}$ immitis were not present, but showed individual, separately walled spherules adjacent to one another. There was no contiguity between adjacent cell walls, and no bud scars were present on the larger cells, as would be found with budding Blastomyces species (Figure 2).

\section{Case 2}

A 51-year-old man presented with right-sided pleuritic chest pain and cough five days after returning from a camping trip to the Grand Canyon (Arizona, USA). He was treated with an oral antibiotic for community-acquired pneumonia, based on his symptoms and an infiltrate on chest radiography. At follow-up, he complained of cough (only occasionally productive of clear sputum) and had no episodes of hemoptysis. He experienced intermittent fevers and chills, but no other systemic complaints or weight loss. He was previously well and on no regular medications. Baseline blood work including complete blood counts, electrolytes, creatinine and liver enzymes were normal.

A repeat chest radiography and high-resolution computed tomography of the chest showed an opacity in the periphery of the right upper lobe. There were no calcifications or cavitations within the lesion. He underwent bronchoscopy, with BAL, mediastinoscopy and biopsy. The organisms seen on the BAL smear were interpreted by a pathologist to be consistent with Blastomyces species. A culture of the specimen grew C immitis.

A three-month treatment with fluconazole $400 \mathrm{mg}$ per day completely resolved the clinical symptoms and radiological findings.

\section{DISCUSSION}

In tissue specimens, detection of mature spherules containing endospores is pathognomonic of coccidioidomycosis and can be identified with a variety of stains (4). Atypical forms of $\mathrm{C}$ immitis in tissues from humans have been recognized in several studies (2). Immature nonendosporulating spherules can

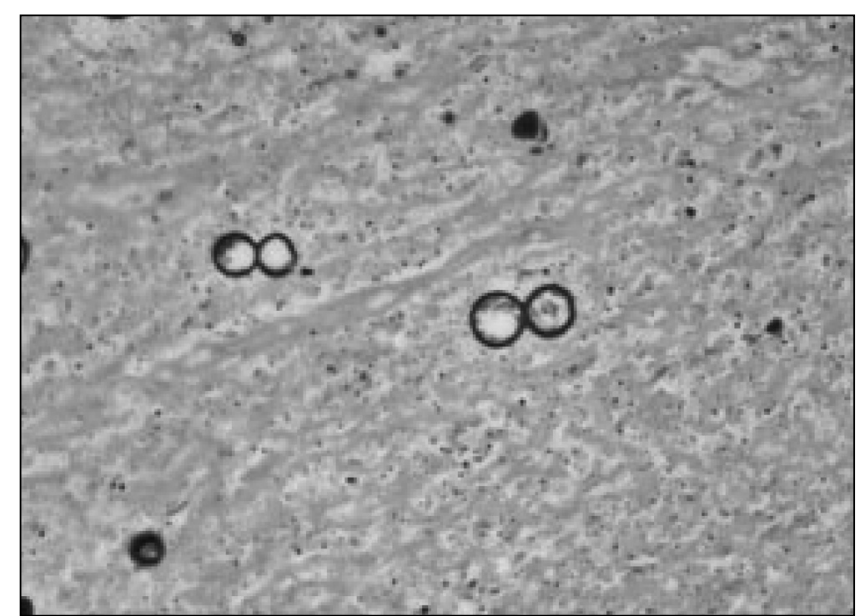

Figure 2) A lung tissue sample containing budding Blastomyces species stained with Gomori methenamine silver

resemble nonbudding forms of Blastomyces dermatitidis (2). Four distinct morphological forms (morula-like, broad-based yeast-like, moniliform and true hyphae) of $\mathrm{C}$ immitis without the typical endospores have been detected and confirmed using fluorescent antibody reagents.

Occasionally, coccidioidomycosis has also been misdiagnosed as carcinoma (4-6). Reactive atypia of pneumocytes is often seen in cytological aspirates, and when spherules are undetected, the reactive atypia may be misidentified as carcinoma (6). Tissue biopsy is superior to cytological apirates for direct visualization of the organism (6). The pitfalls of cytodiagnosis have been highlighted in other reported cases of coccidioidomycosis misdiagnosed as carcinoma (5). Severely reactive bronchial cells can be interpreted as malignant cells because they have cytological features similar to nonsmall cell carcinoma.

In our first patient, it is likely that chronic $\mathrm{C}$ immitis infection contributed to chronic cough and dyspnea. Chronic pulmonary sequelae develop in approximately $5 \%$ of people infected with C immitis, and may occur in individuals who are symptomatic or asymptomatic at the time of primary infection (1). Occasionally, hemoptysis or low-grade fever may occur, as in the present report. Surgical therapy is indicated when there is a rapidly expanding cavity, life-threatening hemoptysis, risk of rupture into the pleural space or recurrent superimposed bacterial infections. Otherwise, lifelong azole suppressive therapy should be administered for chronic pulmonary coccidioidomycosis (6).

\section{CONCLUSION}

Our first patient presented with pulmonary symptoms consistent with pulmonary carcinoma. However, blastomycosis and coccidioidomycosis can present similarly and resemble each other clinically. During the first presentation, cultures for fungi were not performed and the diagnosis was made based on microscopic morphology. Coccidioidomycosis was diagnosed only after culture was performed on lung tissue at follow-up. Because it is not uncommon for spherules to be absent, the morphological forms present were confused with $B$ dermatitidis. In such instances, an examination of the specimen using fluorescent antibody or nucleic acid testing will be diagnostic in the absence of cultures or morphologically typical forms microscopically. 
Our first patient may have had only chronic or recurrent coccidioidomycosis and not carcinoma, or both diseases may have coexisted. The early, correct diagnosis of coccidioidomycosis is important to initiate appropriate therapy, and to prevent unnecessary diagnostic procedures and therapeutic modalities such as lung resection or radiation.

Histopathologists should be aware of atypical forms of Coccidioides species and be cautious in diagnosis of malignancy in the presence of this fungal infection.

CONFLICT OF INTEREST: There are no conflicts of interest for any of the authors.

\section{REFERENCES}

1. Goldman M, Johnson PC, Sarosi GA. Fungal pneumonias. The endemic mycoses. Clin Chest Med 1999;20:507-19.

2. Kaufman L, Valero G, Padhye AA. Misleading manifestations of Coccidiodes immitis in vivo. J Clin Microbiol 1998;36:3721-3.

3. Vaz A, Pineda-Roman M, Thomas AR, Carlson RW. Coccidioidomycosis: An update. Hosp Pract (Minneap) 1998;33:105-8, 113-5, 119-20.

4. Stevens DA. Coccidioidomycosis. N Engl J Med 1995;332:1077-82.

5. Chen KT. Cytodiagnostic pitfalls in pulmonary coccidioidomycosis. Diagn Cytopathol 1995;12:177-80.

6. Case records of the Massachusetts General Hospital. Weekly clinicopathological exercises. Case 35-1981. N Engl J Med 1981;305:507-14. 


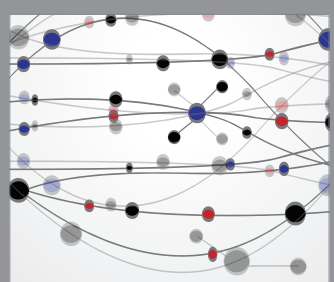

The Scientific World Journal
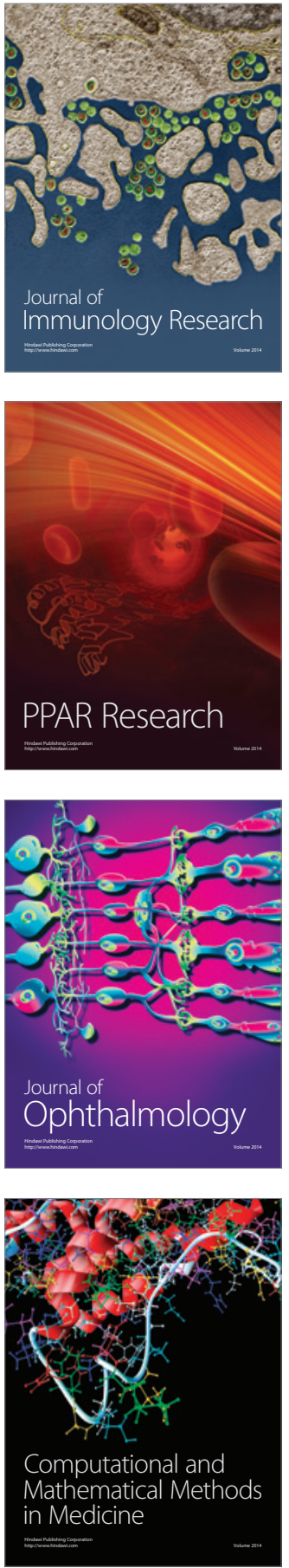

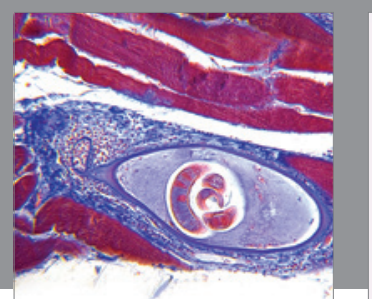

Gastroenterology Research and Practice

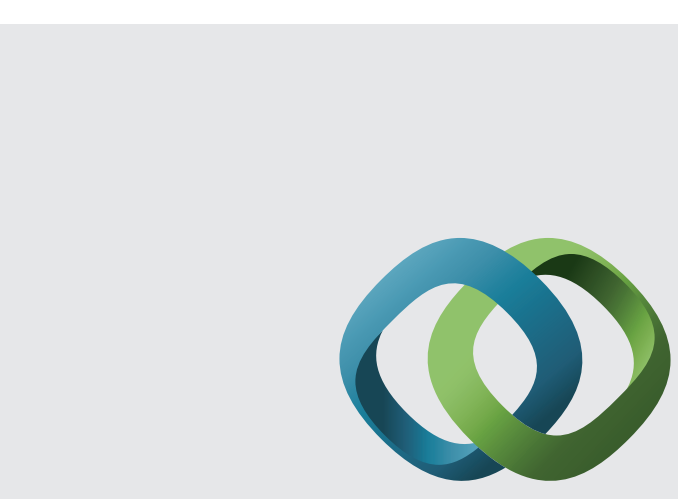

\section{Hindawi}

Submit your manuscripts at

http://www.hindawi.com
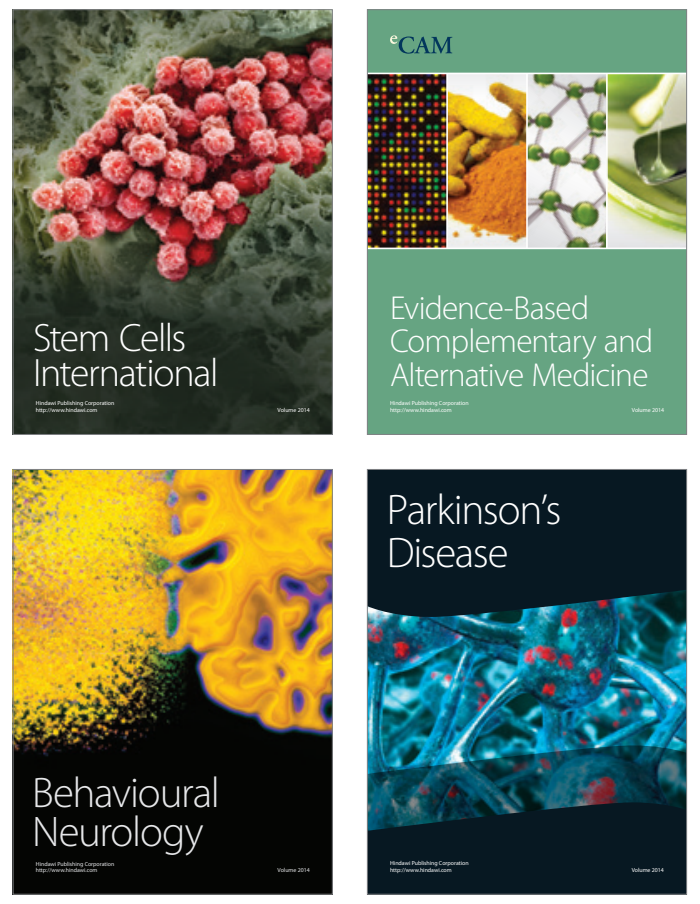
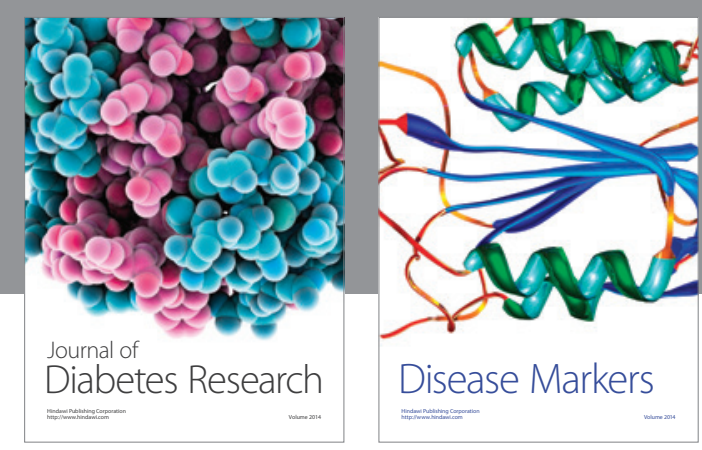

Disease Markers
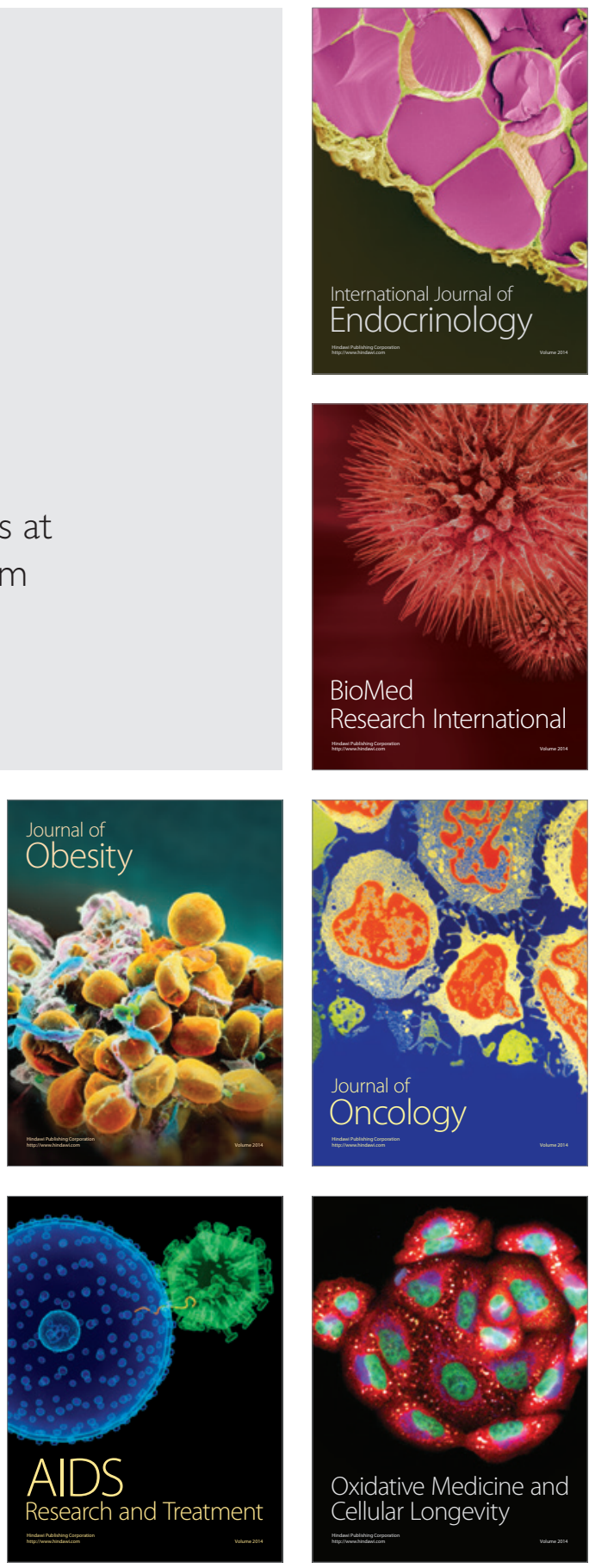